\title{
Efeito do tipo de envase, flatpacks ou palhetas, sobre a criopreservação da fração rica do sêmen asinino
}

[The effect of the type of package, FlatPacks or straws, on cryopreservation of the jackass sperm-rich fraction]

\author{
A.L.R. Sales ${ }^{1}$, A.T. Gonczarowska ${ }^{1}$, L.E. Carvalho $^{1}$, M.S. Palhares ${ }^{2}$,
} R. Rossi ${ }^{1}$, J.M. Silva Filho ${ }^{2 *}$

${ }^{1}$ Aluna de pós-graduação - Escola de Veterinária - Universidade Federal de Minas Gerais - Belo Horizonte, MG ${ }^{2}$ Escola de Veterinária - Universidade Federal de Minas Gerais - Belo Horizonte, MG

\section{RESUMO}

Com o objetivo de se estudar o efeito do tipo de envase sobre a criopreservação do sêmen asinino, coletado de forma fracionada, utilizou-se o sêmen de cinco jumentos da raça Pêga, submetido a um protocolo de congelamento envolvendo um diluidor e duas formas de envase, flatpacks ou palhetas de $0,55 \mathrm{~mL}$, tendo como ponto final a avaliação dos parâmetros seminais in vitro após o descongelamento do sêmen. Os resultados de motilidade espermática para as amostras envasadas em flatpacks ou em palhetas foram de $24,67 \%$ e $35,34 \%$, no momento do descongelamento; de 13,39\% e 25,83\%, 60 minutos após o descongelamento; e de 7,86\% e 13,33\%, aos 120 minutos após o descongelamento, respectivamente. Já os valores para o vigor espermático foram de 2,55 e 3,33, no momento do descongelamento; de 1,57 e 2,48, após 60 minutos do descongelamento; e de 1,11 e 1,67, após 120 minutos do descongelamento, na mesma ordem anterior. As características seminais diferiram entre os jumentos, evidenciando uma grande variação individual. $O$ percentual de ejaculados aprovados (motilidade $\geq 30 \%$, vigor $\geq 3$ ) foi influenciado pelo reprodutor e pelo tipo de envasamento, estando o melhor resultado $(\mathrm{P}<0,05)$, de $86,21 \%$, associado ao sêmen envasado em palhetas, quando comparado ao valor de $56,67 \%$, obtido para o sêmen envasado em flatpacks.

Palavras-chave: jumento, coleta fracionada, sêmen congelado, flatpacks, palhetas

\section{ABSTRACT}

The effect of the type of package on the cryopreservation of jackass semen was studied, using the sperm-rich fraction of five jackasses and a special cryopreservation semen protocol, related to one extender and two different packages, FlatPacks or straws. The characteristics of the in vitro semen were evaluated after thawing. The motility results were $24.67 \%$ and $35.34 \%$ after thawing; $13.39 \%$ and $25.83 \% 60$ minutes after thawing and $7.86 \%$ and $13.33 \%, 120$ minutes after thawing. The vigor results were 2.55 and 3.33 after thawing, 1.57 and 2.4860 minutes after thawing and 1.11 and 1.67120 minutes after thawing, for FlatPacks or straws packages, respectively. The jackasses used were different regarding seminal characteristics, with great individual variation. The approved post-thaw viability (motility $\geq 30 \%$, vigor $\geq 3$ ) was affected by jackass and type of package, with the best results $(P<0.05)$ for semen packed in straws $(86.21 \%)$, compared to semen packed in FlatPacks (56.67\%).

Keywords: jackass, split-ejaculate method, frozen semen, FlatPacks, straws

\section{INTRODUÇÃO}

As biotecnologias da reprodução têm sido ferramentas necessárias ao crescimento da equideocultura mundial. Entre elas, a criopreservação do sêmen é de fundamental

Recebido em 26 de maio de 2014

Aceito em 11 de junho de 2015

*Autor para correspondência (corresponding author)

E-mail: silvafilhojm@gmail.com importância e possui diversas vantagens, como a otimização da utilização do sêmen de reprodutores de alto mérito genético, a constituição de banco genético de animais valiosos, idosos, ou que já vieram a óbito, bem como a prevenção de enfermidades oriundas do trânsito e do contato entre animais. Além disso, 
facilita o armazenamento e o transporte do sêmen, ultrapassando as barreiras geográficas que interferem no transporte do sêmen equídeo (Squires et al., 1999). Entretanto, os altos custos do processo de congelamento, bem como as baixas taxas de gestação resultantes da inseminação artificial com sêmen congelado, têm sido relacionados como pontos negativos, limitando a utilização massal dessa técnica (Cottorello, 2002).

Diante da necessidade real de utilização da inseminação artificial envolvendo o sêmen congelado de equídeos, para o progresso da equideocultura mundial torna-se necessário o desenvolvimento de pesquisas que visem ao aperfeiçoamento de biotecnologias para obtenção de resultados de fertilidade satisfatórios.

O sêmen de equídeos foi congelado, inicialmente, em ampolas de vidro (Polge e Minotakis, 1964). Entretanto, nos trabalhos realizados por Nagase et al. (1966), Oshida (1967) e Merkt et al. (1975), utilizou-se o congelamento em pellets. Como limitantes desse método de congelamento, citam-se as dificuldades de identificação das amostras, bem como a ocorrência de contaminação do sêmen, invibializando seu uso (Davies Morel, 1999).

No final da década de 70, Martin et al. (1979) utilizaram os denominados macrotubos para o envase e o congelamento do sêmen de garanhões, e Tischner (1979), na Polônia, utilizou tubos de alumínio, com o mesmo propósito, em um estudo contemporâneo. Ambos os métodos (macrotubos e os tubos de alumínio) envolvem grandes volumes, o que dificulta o seu armazenamento, além de estarem associados a curvas de resfriamento e de descongelamento inadequadas (Davies Morel, 1999).

A partir da década de 80 , as palhetas francesas de polipropileno, com capacidade de $0,55 \mathrm{~mL}$, foram amplamente utilizadas, sendo este o método de envase mais utilizado até os dias atuais (Amann e Pickett, 1987; Nascimento, 2006). O envase em palhetas apresenta muitas vantagens em relação aos outros métodos, destacando-se a praticidade no momento do envase e a vedação, a identificação fácil e permanente de cada palheta, a facilidade de aquisição desse material e, principalmente, o fato de propiciar o congelamento homogêneo do sêmen (Arruda et al., 1986). Entretanto, ao trabalhar com sêmen de jumentos, Oliveira (2005) não observou diferenças significativas $(\mathrm{P}>0,05)$ de motilidade e vigor espermáticos, quando utilizou palhetas de $0,55 \mathrm{~mL}$ ou macrotubos de $2,5 \mathrm{~mL}$. Por outro lado, Papa et al. (1991) observaram uma motilidade espermática superior nas amostras de sêmen envasadas em palhetas $(36 \%)$ em relação às acondicionadas em macrotubos $(26 \%)$ após a realização do teste de termorresistência.

De acordo com Dell'Aqua Júnior (2000), o sêmen também pode ser armazenado em palhetas de $0,25 \mathrm{~mL}$, embora tenha concluido que as palhetas de $0,25 \mathrm{~mL}$ e as de $0,55 \mathrm{~mL}$ apresentaram resultados similares quanto aos parâmetros de motilidade e vigor espermáticos. Entretanto, o sêmen congelado em palhetas de $0,25 \mathrm{~mL}$ e descongelado a $65^{\circ} \mathrm{C}$ por seis segundos apresentou maior integridade de membrana. As palhetas de pequeno volume, como as de $0,25 \mathrm{~mL}$, são mais finas e possuem maior área de contato, o que proporciona um congelamento mais rápido e mais homogêneo (Squires et al., 1998).

A forma de envase mais utilizada para a espécie suína é o macrotubo de $5 \mathrm{~mL}$ (Minitub, Alemanha). Porém, apresenta um diâmetro de $5,4 \mathrm{~mm}$, o que impede o congelamento e o descongelamento rápidos do sêmen. $\mathrm{Na}$ tentativa de melhorar os resultados físicos e morfológicos do sêmen, bem como os de fertilidade, vários pesquisadores têm utilizado palhetas, de $0,25 \mathrm{~mL}$ e $0,55 \mathrm{~mL}$, para congelar o sêmen suíno (Bwanga et al., 1990). Embora resultados promissores tenham sido obtidos quanto à viabilidade espermática in vitro, tais formas de envasamento têm merecido restrições para o uso prático em suínos, uma vez que é necessário grande número de palhetas para se obter uma dose inseminante.

Nesse contexto, foi desenvolvido um novo método de envase para o sêmen suíno, o flatpack, feito de polietileno, com espessura de $0,2 \mathrm{~mm}$, $30 \mathrm{~cm}$ de comprimento e $22 \mathrm{~mm}$ de largura. $\mathrm{O}$ flatpack possui uma estrutura capaz de permitir congelamento e descongelamento mais rápidos e mais uniformes, quando comparados aos macrotubos (Eriksson e Rodríguez-Martínez, 2000; Saravia et al., 2005).

Saravia (2008) avaliou o congelamento de amostras superconcentradas de sêmen suíno em 
baixo volume $(2 \times 10 \% / \mathrm{mL}$, em flatpacks de $0,7 \mathrm{~mL}$ ) e sua fertilidade, quando da utilização de inseminações intrauterinas profundas em porcas. Como controle, utilizaram-se palhetas de $0,55 \mathrm{~mL}$ e flatpacks com volumes de $5 \mathrm{~mL}$ de capacidade. Embora a motilidade não diferisse entre os tratamentos avaliados, a integridade da membrana plasmática foi mais bem preservada no sêmen envasado em flatpacks de $5 \mathrm{~mL}$ (54\%) e de $0,7 \mathrm{~mL}(49 \%)$, quando comparada à observada nas células envasadas em palhetas de $0,55 \mathrm{~mL}(38 \%)$.

Até o momento, não existem relatos na literatura mundial da utilização do flatpack como alternativa para o envase e congelamento do sêmen de equídeos. Assim, o objetivo deste trabalho foi avaliar o efeito do tipo de envase, flatpack ou palheta, sobre a criopreservação do sêmen asinino, tendo como pontos finais os resultados de motilidade e vigor espermáticos observados após o descongelamento do sêmen e após o teste de termorresistência (TTR) de 120 minutos. Além disso, verificou-se o percentual de partidas de sêmen aprovadas, resultantes das duas formas de envasamento do sêmen.

\section{MATERIAL E MÉTODOS}

O experimento foi realizado no município de Lagoa Dourada, estado de Minas Gerais, Brasil, e compreendeu o intervalo entre os meses de setembro de 2010 e fevereiro de 2011, representando a estação fisiológica de reprodução dos equídeos nessa região (Rossi, 2008).

Foram utilizados cinco jumentos da raça Pêga (Equus asinus), com idade entre cinco e 17 anos e qualidade seminal comprovada após avaliação do desempenho reprodutivo, com base no exame andrológico realizado e nos dados de fertilidade do rebanho, previamente existentes na propriedade.

No presente experimento, adotou-se modelo experimental composto por cinco reprodutores, dois tratamentos (métodos de envase) e três ejaculados por reprodutor, totalizando 15 ejaculados. Cada ejaculado foi submetido a dois tratamentos:

tratamento 1 (E1T1) - ejaculados coletados de forma fracionada, diluídos em diluidor à base de lactose-gema de ovo (Nagase e Niwa, 1964) modificado e envasados em flatpacks (Medical and Device Packaging $\mathrm{AB}^{\circledR}$ ) de $5 \mathrm{~mL}$ para o congelamento $(n=15)$;

tratamento 2 (E1T2) - ejaculados coletados de forma fracionada, diluídos em diluidor à base de lactose-gema de ovo (Nagase e Niwa, 1964) modificado e envasados em palhetas $\left(\mathrm{IMV}^{\circledR}\right)$ de $0,55 \mathrm{~mL}$ para o congelamento $(\mathrm{n}=15)$.

As coletas de sêmen foram realizadas às segundas e às quintas-feiras, no período da manhã, seguindo as recomendações da literatura (Pickett e Amann, 1993). Para a coleta fracionada do sêmen, utilizou-se vagina artificial modelo aberta (Tischner et al., 1974), cujo tamanho foi adaptado para o presente experimento. $\mathrm{O}$ sêmen era recepcionado em um copo coletor plástico de boca larga, de forma a permitir a coleta dos três primeiros jatos do ejaculado (fração rica), evitando-se, assim, possíveis perdas dessa fração.

Após a coleta, as características seminais foram avaliadas, sendo a motilidade expressa em percentual de células móveis/campo $(0-100 \%)$ e o vigor determinado por uma escala subjetiva de 0 a 5, com base na velocidade de deslocamento das células espermáticas nos campos avaliados, em que as células imóveis receberam 0 e aquelas com máxima velocidade de movimentação receberam 5 .

Após a coleta, diluiu-se o sêmen em diluidor à base de lactose-gema de ovo (Nagase e Niwa, 1964), sem glicerol (Tab. 1), na proporção de 1:1 (sêmen:diluidor). Imediatamente após a diluição, colocava-se o tubo com o sêmen em béquer de vidro contendo $500 \mathrm{~mL}$ de água a $37^{\circ} \mathrm{C}$, o qual, a seguir, era transferido para um balcão refrigerador horizontal (Maquinox $\left.{ }^{\circledR}\right)$, regulado para a temperatura de $5^{\circ} \mathrm{C}$. $\mathrm{O}$ recipiente contendo o sêmen permaneceu dentro do balcão refrigerador durante o tempo necessário para que a água do béquer atingisse temperatura de $5-6^{\circ} \mathrm{C}$

No transcorrer do resfriamento, realizava-se o cálculo da concentração espermática, utilizandose contagem celular em câmara de Neubauer. Terminada a contagem, realizava-se o cálculo para obtenção da diluição pretendida, de forma a se obter uma concentração final de $200 \times 10^{6}$ 
espermatozoides móveis/mL de sêmen diluído. Após a realização do cálculo, adicionava-se a quantidade restante de D1 necessária, sendo o D2 adicionado apenas quando a água do béquer alcançava, no interior do balcão, temperatura de
5- $6^{\circ} \mathrm{C}$, obedecendo-se sempre a uma relação de 1:1 (D1:D2) (Tab. 1). Após a diluição final e homogeneização, procedia-se à nova avaliação física do sêmen, como descrito anteriormente.

Tabela 1. Composição do diluidor Nagase e Niwa (1964) modificado, utilizado no congelamento do sêmen asinino

\begin{tabular}{lccc}
\multicolumn{1}{c}{ Ingredientes } & $\mathrm{D} 1 *$ & $\mathrm{D} 2$ & Diluição Final \\
\hline Solução de lactose a 11\% (mL) & 80,00 & 74,27 & 77,14 \\
Gema de ovo (mL) & 20,00 & 18,57 & 19,28 \\
Penicilina G. potássica cristalina (UI) & 100.000 & 100.000 & 100.000 \\
Estreptomicina (g) & 0,10 & 0,10 & 0,10 \\
Orvus es paste (mL) & ----- & 0,16 & 0,08 \\
Glicerol (mL) & ----- & 7,00 & 3,50 \\
Água (q.s.p,mL)** & 100,00 & 100,00 & 100,00 \\
\hline
\end{tabular}

* Nagase e Niwa (1964) modificado.

** Água destilada, deionizada e autoclavada.

A seguir, procedeu-se ao envasamento do sêmen, sendo cada flatpack preenchido com $5 \mathrm{~mL}$ de sêmen diluído, correspondente a uma concentração espermática final de 1 × $10^{9}$ espermatozoides móveis por flatpack. $\mathrm{O}$ restante do sêmen diluído era acondicionado em recipiente de vidro esterilizado, destinado ao envase de palhetas, nas quais se colocou $0,5 \mathrm{~mL}$ de sêmen, com uma concentração final de $100 \mathrm{x}$ $10^{6}$ de espermatozoides móveis, précongelamento, por palheta.

Para o envasamento do sêmen em flatpacks, utilizou-se uma sonda metálica fina com $32 \mathrm{~cm}$ de comprimento e aproximadamente $1 \mathrm{~mm}$ de diâmetro, acoplada a uma seringa plástica com capacidade de $5 \mathrm{~mL}$. A sonda foi introduzida pela abertura existente em uma das extremidades do flatpack, sendo conduzida até a outra extremidade, devidamente lacrada. A partir daí, iniciava-se o seu preenchimento, sendo a sonda retirada lentamente dele, no transcorrer do processo. A seguir, os flatpacks foram lacrados, utilizando-se uma seladora elétrica (Barbi ${ }^{\circledR}$ Maxi 20), colocada dentro do balcão refrigerador, de forma a manter a temperatura do sêmen a 5 $6^{\circ} \mathrm{C}$.

Para o envasamento do sêmen em palhetas, utilizou-se uma bomba de vácuo apropriada (Dia-Pump ${ }^{\circledR}$ Compressor Modelo C - Fanem Ltda. ${ }^{\circledR}$ ), a qual posuía uma mangueira plástica longa, adaptada a um sistema de envasamento com capacidade para 15 palhetas por vez. Assim, a bomba era ativada quando as extremidades livres das palhetas eram colocadas em contato com o sêmen, de forma a preenchê-las até alcançar o lacre presente nas extremidades opostas. As palhetas eram lacradas com álcool polivinílico armazenado em uma placa de Petri, sendo o seu excesso retirado com o uso de papeltoalha. Todo o procedimento de envase foi realizado dentro do balcão refrigerador, procurando-se manter a temperatura entre 5 $6^{\circ} \mathrm{C}$.

Para o pré-congelamento, utilizou-se uma caixa de isopor apropriada, na qual se colocava quantidade de nitrogênio líquido suficiente para atingir $4 \mathrm{~cm}$ de altura, dentro da caixa. As doses de sêmen foram colocadas em bandejas metálicas e posicionadas a $3 \mathrm{~cm}$ de altura do nitrogênio líquido, por período de 15 minutos, quando a caixa de isopor era mantida tampada. A seguir, mergulhavam-se as doses diretamente no nitrogênio para realização do congelamento. Os flatpacks e as palhetas, pós-congelamento, foram transferidos para um botijão contendo nitrogênio líquido, onde ficaram armazenados por, no mínimo, 60 dias antes do descongelamento e da avaliação do sêmen.

O descongelamento dos flatpacks foi realizado em banho-maria, com água na temperatura de $50^{\circ} \mathrm{C}$, por 13 segundos, os quais foram posteriormente transferidos para um banho-maria a $37^{\circ} \mathrm{C}$, por, no mínimo, 20 segundos. Já as palhetas foram descongeladas diretamente no banho-maria a $37^{\circ} \mathrm{C}$, por 30 segundos. 
As avaliações do sêmen pós-descongelamento foram realizadas por dois avaliadores, considerando-se os parâmetros de motilidade, vigor e resistência ao TTR. Realizou-se o TTR do sêmen envasado em palhetas e flatpacks, considerando-se como resultados satisfatórios as amostras que apresentaram motilidade espermática $\geq 30 \%$ e vigor $\geq 3$.

Para melhor visualização das células espermáticas, dificultada pela presença de diluidores à base de gema de ovo, optou-se pela utilização da solução Hanks' Balanced Salt Solution ${ }^{\circledR}$ (Sigma - Aldrich), na quantidade de 500 microlitros para as amostras envasadas em palhetas e de $2,5 \mathrm{~mL}$ para as amostras envasadas em flatpacks, utilizando-se taxa de diluição de 1:1 (volume de sêmen:volume da solução Hanks'). Para realização do TTR, as amostras foram colocadas em banho-maria a $37^{\circ} \mathrm{C}$, e avaliaram-se as características físicas do sêmen (motilidade e vigor espermáticos), aos 60 e 120 minutos após o descongelamento. A motilidade foi expressa em percentual de células móveis/campo $(0-100 \%)$, e o vigor espermático classificado de 0 a 5 , com base na velocidade de deslocamento das células espermáticas nos campos avaliados. As amostras foram avaliadas em um sistema duplo-cego, por dois avaliadores, sendo esse procedimento adotado para todas as avaliações.

O modelo experimental envolveu o efeito do reprodutor $(n=5)$ e do método de envase do sêmen $(n=2)$, bem como a interação entre reprodutor e método de envase, utilizando-se três repetições (três ejaculados por reprodutor), em que foram avaliadas as características físicas do sêmen (motilidade e vigor) e o percentual de ejaculados aprovados.

Os resultados de vigor espermático, mensurados ao longo do processo de congelamento e descongelamento do sêmen, foram analisados pela abordagem não paramétrica, aplicando-se o teste de Kruskal-Wallis (comparação de mais de duas médias) e o teste de Wilcoxon (comparação de duas médias). Os valores percentuais de motilidade espermática foram convertidos em arcosseno $\sqrt{x}_{\mathrm{x}}$, antes da realização das análises estatísticas.
Todas as análises foram processadas pelo programa estatístico Statistical Analysis System, versão 5 - Microsoft®, e o nível de significância adotado foi de $95 \%(\mathrm{P}<0,05)$.

\section{RESULTADOS E DISCUSSÃO}

Em relação ao sêmen fresco, todos os jumentos avaliados apresentaram sêmen de boa qualidade, com valores de motilidade acima de $80 \%$ e vigor maior que 4. Da mesma forma, Rossi (2008) trabalhou com sêmen de jumentos apresentando resultados similares no sêmen in natura (motilidade $>80 \%$; vigor 5 ).

Como não houve diferenças entre os dois avaliadores quanto aos valores de motilidade $(\mathrm{P}>0,05)$ e vigor $(\mathrm{P}>0,05)$ encontrados, os dados foram agrupados, e essa fonte de variação foi excluída dos modelos e das análises estatísticas, os quias envolveram as duas características espermáticas mencionadas (motilidade e vigor).

Assim, os efeitos de jumento e tipo de envasamento sobre a motilidade espermática avaliada, em diferentes períodos após o descongelamento, estão apresentados na Tab. 2, independentemente do avaliador. Observa-se, na Tab. 2, que as amostras de sêmen do jumento 4, quando avaliadas no tempo zero e aos 60 minutos após o descongelamento, apresentaram resultados similares $(\mathrm{P}>0,05)$ para as palhetas $\mathrm{e}$ os flatpacks quanto à motilidade espermática. No entanto, observou-se efeito do tipo de envasamento $(\mathrm{P}<0,05)$ aos 120 minutos após o descongelamento, sendo os valores dos flatpacks inferiores aos das palhetas e flatpacks observados logo após o descongelamento (tempo zero). Além disso, vale salientar que os valores de motilidade observados para as duas formas de envasamento, no tempo de 60 minutos após o descongelamento, e para as palhetas, aos 120 minutos após o descongelamento, foram similares $(\mathrm{P}>0,05)$ entre si, e não diferiram dos demais $(\mathrm{P}>0,05)$, para o jumento 4 .

De uma maneira geral e para os jumentos 1, 2, 3 e 5, não se observou efeito do reprodutor nem da forma de envasamento do sêmen sobre a variável motilidade espermática (Tab. 2) em nenhum dos tempos de avaliação após o descongelamento. 
Efeito do tipo de envase...

Tabela 2. Efeito do jumento e do tipo de envasamento sobre a motilidade espermática avaliada em diferentes períodos de tempo, após o descongelamento do sêmen

\begin{tabular}{ccccccc}
\hline \multirow{2}{*}{ Jumento } & \multicolumn{5}{c}{ Período de tempo após o descongelamento (minutos) } \\
\cline { 2 - 7 } & \multicolumn{2}{c}{0} & \multicolumn{2}{c}{60} \\
\cline { 2 - 7 } & Flatpack & Palheta & Flatpack & Palheta & Flatpack & Palheta \\
\hline 1 & $19,17 \pm 4,29^{\mathrm{a}}$ & $31,67 \pm 4,29^{\mathrm{a}}$ & $12,50 \pm 7,43^{\mathrm{a}}$ & $26,25 \pm 5,25^{\mathrm{a}}$ & $15,00 \pm 7,43^{\mathrm{a}}$ & $21,25 \pm 5,25^{\mathrm{a}}$ \\
2 & $19,17 \pm 4,29^{\mathrm{a}}$ & $28,33 \pm 4,29^{\mathrm{a}}$ & $15,00 \pm 7,43^{\mathrm{a}}$ & $30,00 \pm 5,25^{\mathrm{a}}$ & $12,50 \pm 7,43^{\mathrm{a}}$ & $18,75 \pm 5,25^{\mathrm{a}}$ \\
3 & $30,83 \pm 4,29^{\mathrm{a}}$ & $35,00 \pm 4,29^{\mathrm{a}}$ & $13,13 \pm 5,25^{\mathrm{a}}$ & $20,00 \pm 4,29^{\mathrm{a}}$ & $7,50 \pm 5,25^{\mathrm{a}}$ & $17,50 \pm 4,29^{\mathrm{a}}$ \\
4 & $39,17 \pm 4,29^{\mathrm{a}}$ & $46,67 \pm 4,29^{\mathrm{a}}$ & $17,50 \pm 5,25^{\text {ab }}$ & $21,67 \pm 4,29^{\text {ab }}$ & $3,75 \pm 5,25^{\mathrm{b}}$ & $12,50 \pm 4,29^{\text {ab }}$ \\
5 & $17,00 \pm 4,29^{\mathrm{a}}$ & $30,00 \pm 4,29^{\mathrm{a}}$ & $5,00 \pm 7,43^{\mathrm{a}}$ & $23,75 \pm 5,25^{\mathrm{a}}$ & $5,00 \pm 7,43^{\mathrm{a}}$ & $7,50 \pm 5,25^{\mathrm{a}}$ \\
\hline Total & $24,67 \pm 1,95$ & $35,34 \pm 1,98$ & $13,39 \pm 2,85$ & $25,83 \pm 2,18$ & $7,86 \pm 2,85$ & $13,33 \pm 2,18$ \\
\hline
\end{tabular}

Médias seguidas por letras diferentes na mesma linha diferem entre si $(\mathrm{P}<0,05)$.

Tabela 3. Efeito do jumento e do tipo de envasamento sobre o vigor espermático avaliado em diferentes períodos de tempo, após o descongelamento do sêmen

\begin{tabular}{|c|c|c|c|c|c|c|}
\hline \multirow{3}{*}{ Jumento } & \multicolumn{6}{|c|}{ Período de tempo após o descongelamento (minutos) } \\
\hline & \multicolumn{2}{|c|}{0} & \multicolumn{2}{|c|}{60} & \multicolumn{2}{|c|}{120} \\
\hline & Flatpack & Palheta & Flatpack & Palheta & Flatpack & Palheta \\
\hline 1 & $2,00 \pm 0,34^{\mathrm{bB}}$ & $3,50 \pm 0,34^{\mathrm{aB}}$ & $1,50 \pm 0,58^{\mathrm{abA}}$ & $2,50 \pm 0,41^{\mathrm{bA}}$ & $2,00 \pm 0,58^{\mathrm{abA}}$ & $2,50 \pm 0,41^{\mathrm{bA}}$ \\
\hline 2 & $1,75 \pm 0,34^{\mathrm{aB}}$ & $2,67 \pm 0,34^{\mathrm{aC}}$ & $2,00 \pm 0,58^{\mathrm{aA}}$ & $2,50 \pm 0,41^{\mathrm{aA}}$ & $1,25 \pm 0,58^{\mathrm{aA}}$ & $1,50 \pm 0,41^{\mathrm{aA}}$ \\
\hline 3 & $2,83 \pm 0,34^{\mathrm{abB}}$ & $3,25 \pm 0,34^{\mathrm{aBC}}$ & $1,63 \pm 0,41^{\mathrm{cdA}}$ & $2,00 \pm 0,34^{\mathrm{abcdA}}$ & $1,25 \pm 0,41^{\mathrm{dA}}$ & $2,25 \pm 0,34^{\mathrm{bcA}}$ \\
\hline 4 & $3,67 \pm 0,34^{\mathrm{bA}}$ & $4,33 \pm 0,34^{\mathrm{aA}}$ & $1,63 \pm 0,41^{\mathrm{cA}}$ & $2,33 \pm 0,34^{\mathrm{cA}}$ & $0,50 \pm 0,41^{\mathrm{cA}}$ & $1,83 \pm 0,34^{\mathrm{cA}}$ \\
\hline 5 & $2,60 \pm 0,37^{\mathrm{aB}}$ & $2,67 \pm 0,34^{\mathrm{abBC}}$ & $1,00 \pm 0,58^{\mathrm{abA}}$ & $2,13 \pm 0,41^{\mathrm{abA}}$ & $1,00 \pm 0,58^{\mathrm{abA}}$ & $1,13 \pm 0,41^{\mathrm{bA}}$ \\
\hline Total & $2,55 \pm 0,16$ & $3,33 \pm 0,16$ & $1,57 \pm 0,23$ & $2,48 \pm 0,18$ & $1,11 \pm 0,23$ & $1,67 \pm 0,18$ \\
\hline
\end{tabular}

Médias seguidas por letras diferentes na mesma linha diferem entre si $(\mathrm{P}<0,05)$.

Médias seguidas por letras diferentes na mesma coluna diferem entre si $(\mathrm{P}<0,05)$.

$\mathrm{Na}$ Tab. 3, estão apresentados os dados referentes ao vigor espermático, podendo-se observar influência do jumento e do tipo de envasamento sobre essa variável, bem como a interação jumento $x$ tipo de envasamento.

Assim, o vigor espermático foi influenciado $(\mathrm{P}<0,05)$ pelos jumentos apenas no tempo zero e envolveu os dois sistemas de envasamento do sêmen (Tab. 3). Observa-se que o jumento 4 apresentou o maior vigor espermático, diferindo $(\mathrm{P}<0,05)$ dos demais jumentos (J1, J2, J3 e J5) nos dois tipos de envasamento. No tempo zero, após o descongelamento, não se observou influência $(\mathrm{P}>0,05)$ do tipo de envasamento sobre o vigor espermático para os jumentos 2,3 e 5. Entretanto, observou-se superioridade das amostras envasadas em palhetas $(\mathrm{P}<0,05) \mathrm{em}$ relação aos flatpacks no sêmen dos jumentos $1 \mathrm{e}$ 4. Aos 60 minutos após o descongelamento, não se observou efeito do jumento nem do tipo de envasamento $(\mathrm{P}>0,05)$ sobre $\mathrm{o}$ vigor espermático. O mesmo resultado foi observado quando da avaliação do sêmen, após 120 minutos do descongelamento, com exceção do jumento 3 , quando os valores de vigor, inerentes às palhetas, superaram $(\mathrm{P}<0,05)$ os obtidos pelas células espermáticas congeladas em flatpacks.

Considerando-se como satisfatórias as partidas de sêmen que apresentaram um mínimo de $30 \%$ de motilidade e vigor $\geq 3$ (Pickett et al., 1987), observou-se que as amostras envasadas em palhetas de $0,55 \mathrm{~mL}$ apresentaram melhor viabilidade espermática. Assim, ao se analisarem os valores de motilidade e vigor espermáticos das amostras envasadas nesse sistema no momento do descongelamento do sêmen, observou-se que apenas um (J2) dos cinco reprodutores não apresentou valores de motilidade espermática satisfatórios (Tab. 2), enquanto dois dos cinco não apresentaram valores de vigor espermático aceitáveis (J2 e J5 Tab. 3 ). Em relação ao flatpack, apenas dois jumentos (J3 e J4) apresentaram valores de motilidade aceitáveis (Tab. 2). No que se refere 
ao vigor, apenas um jumento (J4) apresentou resultados aceitáveis, dentro do limite mínimo descrito anteriormente (Tab. 3).

Quando observadas após 60 e 120 minutos do descongelamento, todas as amostras apresentaram valores de motilidade e de vigor inferiores ao mínimo aceitável, com exceção do jumento 2, que apresentou, aos 60 minutos após o descongelamento, motilidade de 30,00 $\pm 5,25$ para o envasamento em palheta.

Os resultados obtidos, no presente experimento, discordam dos descritos por Silva et al. (1997), quando se observaram valores similares de motilidade e de vigor espermáticos, após a realização do TTR, para as amostras de sêmen asinino envasadas em macrotubo $(4 \mathrm{~mL})$ ou em tubos de alumínio (10mL). Samper et al. (1994) observaram taxas de gestação similares, ao utilizarem palhetas $(64,3 \%)$ ou macrotubos $(59,8 \%)$, para o congelamento do sêmen de garanhões. Da mesma forma, ao comparar dois tipos de envasamento para o congelamento de sêmen asinino (macrotubos de $2,5 \mathrm{~mL}$ e palhetas de $0,55 \mathrm{~mL}$ ), Oliveira (2005) não observou diferenças estatísticas entre eles no que diz respeito às variáveis de motilidade e vigor espermáticos avaliadas. Saravia (2008) trabalhou com sêmen congelado de suínos e observou que o sistema de envasamento não influenciou a motilidade espermática; no entanto, a integridade da membrana plasmática foi mais bem preservada no sêmen envasado em flatpacks de
$5 \mathrm{~mL}(54 \%)$ e de $0,7 \mathrm{~mL}(49 \%)$ em relação às células envasadas em palhetas de $0,55 \mathrm{~mL}$ (38\%).

Diferentemente dos estudos mencionados anteriormente, vários autores, ao trabalharem com o sêmen congelado de equinos, observaram diferenças estatísticas entre alguns tipos de envasamento. Nesse sentido, Papa et al. (1991) observaram que a motilidade espermática das amostras de sêmen envasadas em palhetas foi superior à das acondicionadas em macrotubos, com valores de $36 \%$ e $26 \%$, respectivamente, após a realização do TTR. Heitland et al. (1996) também descreveram melhores resultados para o sêmen envasado em palhetas (menores volumes), após o descongelamento, em comparação aos macrotubos (maiores volumes). Ainda, Dell'Aqua Júnior (2000) observou melhores resultados $(\mathrm{P}<0,05)$ de motilidade e de vigor espermáticos nas amostras de sêmen envasadas em palhetas de $0,55 \mathrm{~mL}$ e de $0,25 \mathrm{~mL}$, quando estas foram comparadas àquelas envasadas em macrotubos de $4 \mathrm{~mL}$, após o descongelamento.

Os melhores resultados associados às palhetas, quanto à manutenção da viabilidade espermática, reforçam a sua importância como a forma de envasamento mais empregada para o congelamento do sêmen de asininos (Arruda et al., 1986; Papa et al., 1991).

O efeito do sistema de envasamento sobre o percentual de ejaculados aprovados após o descongelamento do sêmen está apresentado na Tab. 4.

Tabela 4. Efeito do sistema de envasamento sobre o percentual de ejaculados aprovados após o descongelamento, considerando-se a motilidade $(\geq 30 \%)$ e o vigor $(\geq 3)$ isoladamente ou agrupados

\begin{tabular}{cccc}
\hline $\begin{array}{c}\text { Sistema de } \\
\text { envasamento }\end{array}$ & Motilidade $\geq 30 \%$ & Vigor $\geq 3$ & $\begin{array}{c}\text { Motilidade } \geq 30 \% \\
\text { Vigor } \geq 3\end{array}$ \\
\hline Flatpack & $18 / 30(60,00 \%)^{\mathrm{B}}$ & $18 / 30(60,00 \%)^{\mathrm{B}}$ & $17 / 30(56,67 \%)^{\mathrm{B}}$ \\
Palheta & $25 / 29(86,21 \%)^{\mathrm{A}}$ & $26 / 29(89,66 \%)^{\mathrm{A}}$ & $25 / 29(86,21 \%)^{\mathrm{A}}$ \\
\hline TOTAL & $43 / 59(72,88 \%)$ & $44 / 59(74,58 \%)$ & $42 / 59(71,19 \%)$ \\
\hline
\end{tabular}

Números seguidos por letras diferentes na coluna diferem entre si $(\mathrm{P}>0,05)$.

Há que se salientar (vide Tab.4) a influência $(\mathrm{P}<0,05)$ do sistema de envasamento sobre $\mathrm{o}$ percentual de ejaculados aprovados, seguindo metodologia proposta po Pickett et al. (1987), sendo os melhores resultados $(\mathrm{P}<0,05)$ obtidos para o sêmen envasado em palhetas em comparação aos observados para o envasamento em flatpacks $(86,21$ vs. 56,67, respectivamente -
$\mathrm{P}<0,05)$. Tais resultados estão de acordo com os apresentados por Papa et al. (1991), Heitland et al. (1996) e Dell'Aqua Júnior (2000), que também observaram melhores resultados, após o descongelamento do sêmen, associados à utilização de palhetas. Entretanto, discordam dos resultados apresentados por Samper et al. (1994), Silva et al. (1997) e Oliveira (2005), que não 
observaram diferenças estatísticas entre os diferentes métodos de envasamento avaliados.

\section{CONCLUSÕES}

Diante do alto percentual de ejaculados aprovados após o descongelamento, o sistema de envasamento em palhetas continua sendo o método de escolha para o congelamento do sêmen de equídeos.

\section{REFERÊNCIAS}

AMANN, R.P.; PICKETT, B.W. Principle of cryopreservation and a review of cryopreservation of stallion spermatozoa. J. Equine Vet. Sci., v.7, p.145173, 1987.

ARRUDA, R.P.; VIEIRA, R.C.; MANZANO, A. Inseminação artificial de equídeos com sêmen congelado em palhetas de $0.5 \mathrm{~mL}$. In: CONGRESSO BRASILEIRO DE MEDICINA VETERINÁRIA, 10., 1986, Cuiabá. Anais... Cuiabá: Universidade Federal de Mato Grosso, 1986. p.181.

BWANGA, C.O.; BRAGANCA, M.; EINARSSON, S. et al. Freezing of boar semen in mini- and maxistraws. J. Vet. Med. Assoc., v.37, p.651-658, 1990.

COTTORELlO, A.C.P. Criopreservação de sêmen equino utilizando associação de etilenoglicol e glicerol. 2002. 47f. Dissertação (Mestrado em Ciência Animal) - Escola de Veterinária, Universidade Federal de Minas Gerais, Belo Horizonte, MG.

DAVIES MOREL, M.C.G. Equine artificial insemination. Wallingford-Oxon: CAB International, 1999, 406p.

DELL'AQUA JÚNIOR, J.A. Efeito da centrifugação, tipos de envase e temperatura de descongelação sobre os parâmetros espermáticos e índices de fertilidade relacionados com o local de deposição e concentração da dose inseminante do sêmen congelado equino. 2000. 81f. Dissertação (Mestrado) - Faculdade de Medicina Veterinária e Zootecnia, Universidade Estadual Paulista, Botucatu, SP.

ERIKSSON, B.M.; RODRIGUEZ-MARTINEZ, H. Effect of freezing and thawing rates on the post-thaw viability of boar spermatozoa frozen in FlatPacks and Maxi-straws. Anim. Reprod. Sci., v.63, p.205-220, 2000.

HEITLAND, A.V.; JASKO, D.J.; SQUIRES E.L. et $a l$. Factors affecting motion characteristics of frozenthawed stallion spermatozoa. Equine Vet. J., v.28, p.47-53, 1996.
MARTIN, J.C.; KLUG, E.; GUNZEL, A.R. Centrifugation of stallion semen and its storage in large volume straws. J. Reprod. Fertil., suppl.27, p.4751,1979 .

MERKT, H.; KLUG, E.; KRAUSE, D. et al. Results of long-term storage of stallion semen frozen by the pellet method. J. Reprod. Fertil, suppl.23, p.105-106, 1975.

NAGASE, H.; NIWA, T. Deep freezing bull semen in concentrated pellet form. I. Factors affecting survival of spermatozoa. In: INTERNATIONAL CONGRESS ANIMAL REPRODUCTION AND ARTIFICIAL INSEMINATION, 5., 1964, Trento. Proceedings.... Trento, Italy: ICAR 1964. p.410-415.

NAGASE, H.; SOEJIMA, A.; NIWA, T. et al. Studies on the freezing storage of stallion semen. I. Fertility results of stallion frozen semen in concentrated pellet form. J. Anim. Reprod., v.12, p.48-51, 1966.

NASCIMENTO, J. Efeitos da concentração espermática e volume sobre as características do movimento espermático (CASA) e sobre membranas plasmática, acrossomal e mitocondrial (microscopia e epifluorescência) de espermatozoides equinos criopreservados. 2006. 107f. Dissertação (Mestrado em Engenharia de Alimentos) - Faculdade de Zootecnia e Engenharia de Alimentos, Universidade de São Paulo, Pirassununga, SP.

OLIVEIRA, J.V. Estudo de metodologias para a criopreservação de sêmen de jumento (Equus asinus) por meio de testes laboratoriais e fertilidade. 110f. 2005. Dissertação (Mestrado em Medicina Veterinária) - Faculdade de Medicina Veterinária e Zootecnia, Universidade Estadual Paulista "Julio de Mesquita Filho", Botucatu, SP.

OSHIDA, H. Studies on frozen stallion semen III. Jpn. J. Anim. Reprod., v.13, p.136-140, 1967.

PAPA, F.O.; CAMPOS FILHO, E.P.; ALVARENGA, M.A. Influência da forma de acondicionamento sobre a integridade acrossômica e termo-resistência do sêmen congelado equino. In: CONGRESSO BRASILEIRO DE REPRODUÇÃO ANIMAL, 9., 1991, Belo Horizonte-MG. Proceedings.... Belo Horizonte: CBRA, 1991, p.456.

PICKETT, B.W.; AMANN, R.P. Cryopreservation of Semen. In: MCKINNON, A.O.E; VOSS, J. L. Equine reproduction, Malvern: Lea \& Febiger, 1993. p.769789.

PICKETT, B.W.; SQUIRES, E.L.; McKINNON, A.O. et al. Procedures for collection, evaluation and utilization of stallion semen for artificial insemination. Fort Collins: Colorado State University Animal Reproduction Laboratory, 1987. 125p. (Bulletin, 03). 
POLGE, C.; MINOTAKIS, C. Deep freezing of jackass and stallion semen. In: V INTERNATIONAL CONGRESS ANIMAL REPRODUCTION AND ARTIFICIAL INSEMINATION, 5., 1964, Trento. Proceedings... Trento, Italy: ICAR 1964. p.545-552.

ROSSI, R. Comparação de dois diluidores na fertilidade de éguas inseminadas com sêmen asinino a fresco ou resfriado. 2008. 209f. Dissertação (Mestrado em Medicina Veterinária) - Escola de Veterinária, Universidade Federal de Minas Gerais, Belo Horizonte, MG.

SAMPER, J.C.; HEARN, P.; GANHEIM, A.E. et al. Pregnancy rates and effect of extender and motility and acrosome status of frozen-thawed stallion spermatozoa. In: ANNUAL CONVENTION OF THE AMERICAN ASSOCIATION OF EQUINE PRACTITIONERS, 40., 1994, Vancouver. Proceedings... Vancouver: American Association of Equine Practitioners, 1994. p.41-43.

SARAVIA, F.; WALLGREN, M.; NAGY, S. et al. Deep freezing of concentrated boar semen for intrauterine insemination: effects on sperm viability. Theriogenology, v.63, p.1320-1333, 2005.
SARAVIA, F. Cryopreservation of boar semen. 2008. 63f. Thesis (Doctoral) - Faculty of Veterinary Medicine and Animal Science, Swedish University of Agricultural Sciences, Uppsala.

SILVA, S.S.; HENRY, M.; NUNES, A.S. et al. Influência do sistema de envasamento sobre a qualidade espermática de jumentos (Equus asinus) avaliada in vitro pós-descongelação. Rev. Bras. Reprod. Anim., v.21, p.140-146, 1997.

SQUIRES, E.L.; BRUBAKER, J.K.; MC CUE, P.M. et al. Effect of sperm number and frequency of insemination on fertility of mares inseminated with cooled semen. Theriogenology, v.49, p.743-749, 1998.

TISCHNER, M. Evaluation of deep-frozen semen in stallions. J. Reprod. Fertil., Suppl.27, p.53-59, 1979.

TISCHNER, M.; KOSINIAK, K.; BIELÁNSKI, W. Analysis of the pattern of ejaculation in stallion. $J$. Reprod. Fertil., v.41, p.329-335, 1974. 